Unfallchirurg 2017 $120: 94-95$

DOI 10.1007/s00113-016-0303-0

C) Springer Medizin Verlag Berlin 2017

CrossMark

\author{
W. Mutschler ${ }^{1} \cdot$ D. Höntzsch ${ }^{2}$ \\ 'Klinik für Allgemeine, Unfall-, Hand- und Plastische Chirurgie, München, Deutschland \\ ${ }^{2}$ Tübingen, Deutschland
}

\title{
Stahl oder Titan in der Osteosynthese
}

Stahl oder Titan, was für ein Thema in Zeiten, in denen in deutschsprachigen und nordeuropäischen Ländern überwiegend Titan als Werkstoff für Osteosynthesen verwendet und Stahl als Reservematerial oder für bestimmte Lokalisationen wie dem Becken vorgehalten werden. Titan und für manches Stahl ist die bei uns verfolgte und lange nicht mehr hinterfragte Devise.

Das war in den 1980er-Jahren anders. Bis dato gab es nur Implantate aus Stahl; Titan entwickelte sich erst allmählich technologisch und preislich zum Konkurrenten von Stahl. Maßgeblich dafür waren einerseits klinische Untersuchungen, die auf eine höhere Allergierate bei Stahlimplantaten verwiesen und andererseits experimentelle Untersuchungen, die Titan als Werkstoff mit geringerer Infektanfälligkeit und günstigerem Verhalten im eingetretenen Infektfall sahen. Stahl oder Titan wurde im Interesse der Patientensicherheit zugunsten von Titan entschieden.

In den USA wurde dieser Wechsel nicht vollzogen. Dort sind bis heute und werden wohl auch in Zukunft mehrheitlich Platten und Schrauben aus Stahl verwendet. Neben Optimierungen der Stahllegierung waren dafür auch Erfahrungen und Berichte über technische Schwierigkeiten im Umgang mit Titanimplantaten, v. a. mit winkelstabilen Platten und Schrauben und besonders bei Metallentfernungen, von Bedeutung.

Die Technische Kommission der Arbeitsgemeinschaft Osteosynthese griff diese Anwendungsdiskrepanz auf und wurde durch nationale und internationale Experten unterstützt, die in Tübingen im Oktober 2016 abschließend diskutierten. Anlass für uns, das Thema auch für die Leserinnen und Leser unserer Zeitschrift in 4 Beiträgen von ausgewiesenen Kennern der Materie aufbereiten zu lassen.

Die Arbeitsgruppe von Duda arbeitet heraus, dass Stahl- und Titanlegierungen eine ähnliche Bruch- und Dauerfestigkeit aufweisen und somit vergleichbare Eigenschaften unter statischer und zyklischer Belastung aufweisen. Das Hauptunterscheidungsmerkmal ist die deutlich höhere Elastizität von Titan, was bei Belastung und gleicher Konfiguration der Osteosynthese eine entsprechend höhere interfragmentäre Bewegung bei Plattenosteosynthesen erlaubt. Wenn eine solche also gewünscht ist, wären Titanimplantate vorzuziehen. Dies gilt allerdings nur für die frühe Phase der sekundären Frakturheilung, danach spielen Plattendesign, Schraubenanzahl und -position eine deutlich größere Rolle. Und es gilt auch, dass die Frakturkonfiguration und die Art der Belastung bedeutendere Einflussgrößen für die Frakturheilung darstellen.

Die systematische Literaturrecherche von Joeris et al. ergab zunächst, dass es erstaunlich wenig vergleichende Studien mit hoher Evidenz zu diesem Thema gibt. Letztlich war in den analysierten klinischen Studien kein signifikanter Unterschied hinsichtlich Frakturheilung, Allergie-und Infektraten oder mechanischen Problemen zwischen den beiden Materialien auszumachen. Das angesprochene praktische Problem der manchmal erschwerten Materialentfernung bei Titan wurde bisher nicht systematisch thematisiert. Die angenommene Überlegenheit von Titan gegenüber Stahl wurde, wie bereits oben erwähnt, überwiegend mit der geringeren Korrosionsan- 
fälligkeit, der besseren Biokompatibilität und einer geringeren Infektanfälligkeit, gemessen am Ausmaß der Fremdkörperreaktion, der allergischen Reaktionshäufigkeit und der Infektrate begründet. Da die systematische Literaturrecherche aus dem genannten Grund der unzureichenden Evidenz hierzu wenig beiträgt, beschäftigen sich 2 Beiträge explizit mit dieser Problematik.

Haubruck und Schmidmaier fanden keinen wirklichen Vorteil für Titan hinsichtlich einer geringeren Rate an periund postoperativen implantatassoziierten Infektionen.

Thomsen und Peter berichten über Einzelfällen von allergischen Reaktionen auf heutige Stahlimplantate und sehen die Verwendung von Stahl in der Summe als unkritisch an; es sei denn, es liegt eine präoperativ bekannte Nickelallergie vor. In solchen Fällen sollten Titanimplantate verwendet werden, v. a. wenn diese direkt unter der Haut zu liegen kommen.

Stahl und Titan können somit, mit Ausnahme der Metallallergie, nach heutiger Evidenz als gleichwertig angesehen werden. Was bedeutet das für die tägliche Praxis?

Das Dogma der Minderwertigkeit von Stahlimplantaten zur Frakturversorgung, schon 2012 von Weckbach et al. in Der Unfallchirurg infrage gestellt, lässt sich nicht länger aufrechterhalten. Weichteilschaden, Knochenqualität, Frakturkonfiguration und eine adäquate Operationstechnik sind für eine sichere Frakturheilung weitaus bestimmendere Einflussfaktoren als das gewählte Material. Abzuwägen sind im Einzelfall die intraoperative Handhabung der jeweiligen Implantate aus Stahl bzw. Titan und mögliche Probleme bei der Materialentfernung. Weiterhin ist sicherlich der Patientenwunsch zu berücksichtigen und $\mathrm{zu}$ dokumentieren. Bei bekannter Metallallergie ist die einhellige Meinung, Titanimplantate $\mathrm{zu}$ verwenden.

Wir danken den Autoren dieses Leitthemas für ihre Beiträge und hoffen, bei unserer Leserschaft die Diskussion anzuregen.

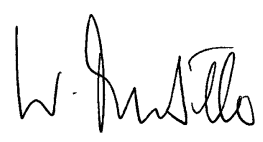

Prof. Dr. W. Mutschler

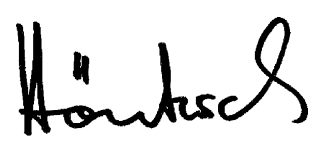

Prof. Dr. D. Höntzsch

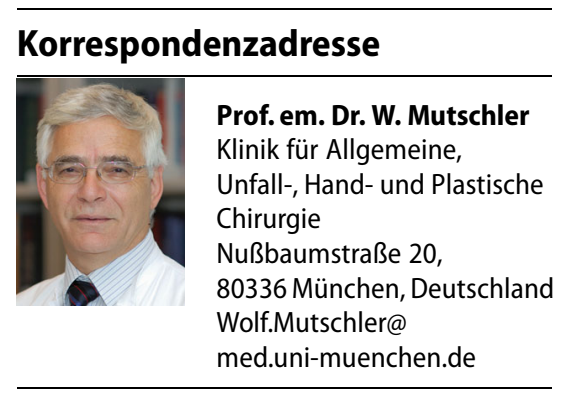

Interessenkonflikt. W. Mutschler und D. Höntzsch geben an, dass kein Interessenkonflikt besteht.

\section{B. Jenny \\ Projektmanagement}

Das Wissen für eine erfolgreiche Karriere

Zürich: vdf Hochschulverlag AG an der ETH Zürich 2016, 5. Auflage, 416 S., (ISBN: 978-3-7281-3766-1), gebunden 74,90 EUR

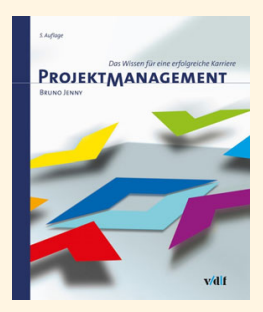

Die Einordnung von Aufgaben in Projekte ist ein Grundpfeiler moderner Unternehmen aller Branchen. Doch solche Projekte stehen

und fallen mit der Kompetenz der Projektleitung. Um also einen Projekterfolg zu garantieren, bedarf es eines fachkundigen, durchorganisierten Projektmanagements. Projektmanagement - Das Wissen für eine erfolgreiche Karriere des schweizer Unternehmensberaters, Dozenten und Prüfungsexperten Bruno Jenny veranschaulicht in verständlicher und gut strukturierter Weise, wie sich ein Managementsystem optimieren lässt. Jenny zeigt beispielsweise den Mehrwert eines psychologisch ausgerichteten Change Managements im Vergleich zur konventionellen Projektabwicklung auf und demonstriert zudem, warum die optimale Interaktion der einzelnen Projektelemente unerlässlich ist. Anhand von einleuchtenden Lernzielen, Aufgaben, Musterlösungen und vielen Grafiken lehrt es den Leser das vielschichtige moderne Projektmanagement.

\section{D.A. Groneberg (Frankfurt am Main)}

